\title{
Geometry of exceptional super Yang-Mills theories
}

\author{
Michael Rios* \\ Dyonica ICMQG, Los Angeles, 90032 California, USA \\ Alessio Marrani ${ }^{\dagger}$ \\ Museo Storico della Fisica e Centro Studi e Ricerche Enrico Fermi, \\ Via Panisperna 89A, I-00184 Roma, Italy \\ David Chester \\ Department of Physics and Astronomy, UCLA, Los Angeles, 90024 California, USA
}

(Received 26 November 2018; published 13 February 2019)

\begin{abstract}
Some time ago, Bars found $D=11+3$ supersymmetry and Sezgin proposed super Yang-Mills theory (SYM) in $D=11+3$. Using the "magic star" projection of $\mathfrak{e}_{8(-24)}$, we show that the geometric structure of SYM's in $12+4$ and $11+3$ space-time dimensions descends to the affine symmetry of the space $\mathrm{AdS}_{4} \otimes S^{8}$. By reducing to transverse transformations along maximal embeddings, the near-horizon geometries of the M2 brane $\left(\operatorname{AdS}_{4} \otimes S^{7}\right)$ and M5 brane $\left(\operatorname{AdS}_{7} \otimes S^{4}\right)$ of M-theory are recovered. Utilizing the recently introduced "exceptional periodicity" (EP) and exploiting the embedding of semisimple rank-3 Jordan algebras into rank-3 T-algebras of special type yields the spaces $\mathrm{AdS}_{4} \otimes S^{8 n}$ and $\operatorname{AdS}_{8 n-1} \otimes S^{5}$ with reduced subspaces $\mathrm{AdS}_{4} \otimes S^{8 n-1}$ and $\mathrm{AdS}_{8 n-1} \otimes S^{4}$, respectively. As such, EP describes the nearhorizon geometries of an infinite class of novel exceptional SYM's in $(8 n+3)+3$ dimensions that generalize M-theory for $n=1$. Remarkably, the $n=3$ level hints at M2 and M21 branes as solutions of bosonic M-theory and gives support for Witten's monstrous AdS/CFT construction.
\end{abstract}

DOI: 10.1103/PhysRevD.99.046004

\section{INTRODUCTION}

After Witten proposed M-theory [1], arguments to go beyond $D=11$ have been put forth by Vafa [2], Bars [3,4], Sezgin [5], and Nishino [6]. Bars found success in his model of S-theory with two time dimensions in $D=$ $s+t=11+2[3]$ and proposed $D=11+3$ supersymmetry with three times [4], while Sezgin formulated super Yang-Mills theories (SYM's) up to signature $11+3$ [5]. The signature $11+3$ also found promise in graviGUT models $[7,8]$. Nishino pushed beyond $D=$ $11+3$ and gave arguments for SYM's in signature $(9+m)+(1+m)$, for arbitrary $m \in \mathbb{N} \cup\{0\}$.

The symmetry of $11+3$ space-time, with a 64dimensional Majorana-Weyl (MW) spinor, interestingly arises in a certain 5-grading of "extended Poincaré type"

*mrios@dyonicatech.com

jazzphyzz@gmail.com

dchester@ucla.edu

Published by the American Physical Society under the terms of the Creative Commons Attribution 4.0 International license. Further distribution of this work must maintain attribution to the author(s) and the published article's title, journal citation, and DOI. Funded by SCOAP ${ }^{3}$. of $\mathfrak{e}_{8(-24)}$, while the symmetry of $10+2$ space-time is seen in a 5-grading of "contact type" of $\mathfrak{e}_{7(-25)}$, with a 32-dimensional MW spinor [9]. Moreover, a certain 3 -grading of $\mathfrak{e}_{6(-26)}$ contains the symmetry of $9+1$ spacetime with a 16-dimensional MW spinor. These are precisely the signatures studied by Bars and Sezgin up to $11+3[4,5]$.

Through a projection of $\mathfrak{e}_{8(-24)}$ along an $\mathfrak{S I}_{3, \mathbb{R}}$ subalgebra (the so-called magic star projection, cf. Fig. 1), the hidden Jordan algebraic structure within $\mathfrak{e}_{8(-24)}$ becomes manifest [10-13]. The central vertex $\mathfrak{e}_{6(-26)}$ of the projection then encodes the reduced structure symmetry of the degree three exceptional Jordan algebra (also known as Albert algebra) $\mathbf{J}_{3}^{\bigotimes}$, mapped to six vertices of the star projection.

Using the aforementioned gradings of $\mathfrak{e}_{6(-26)}, \mathfrak{e}_{7(-25)}$, and $\mathfrak{e}_{8(-24)}$, as well as the magic star, a periodic extension of the exceptional Lie algebras can be formulated. This periodic extension, dubbed exceptional periodicity (EP) [13], allows higher dimensional extensions of the exceptional Lie algebras, while also permitting arbitrarily high dimensional magic star projections. Although the resulting algebras (with generalized roots) no longer satisfy the Jacobi relation, they do contain Lie algebraic reductive parts, as well as a normalized cocycle, as seen in lattice vertex algebras $[13,14]$.

In this study, using some gradings of the exceptional Lie algebras and their exceptionally periodic extensions, we 
show that the geometric structure of SYM's in $11+3$ and $12+4$ space-time dimensions can be recovered from the affine symmetry of the space $\mathrm{AdS}_{4} \otimes S^{8}$, with the 8-sphere being a line in the Cayley plane $\mathbb{O P}^{2}$, the space of rank-1 projectors of $J_{3}^{\mathbb{Q}}[15,16]$. The symmetry of $S^{8}, S O(9)$ (the lightcone little group of M-theory), is a maximal and symmetric subgroup of $F_{4}$, and as such is the stabilizer of $\mathbb{O P}^{2} \simeq F_{4} / S O(9)$ itself. A fixed point of $\mathbb{O P}^{2}$, a rank-1 idempotent of $J_{3}^{\mathbb{Q}}$, identifies one of three possible embeddings $S O(9) \subset F_{4}$ and spans an orthogonal direction that can serve as the 11th dimension of M-theory. By considering transverse directions along maximal embeddings, the near-horizon geometries of the M2 brane $\left(\mathrm{AdS}_{4} \otimes S^{7}\right)$ and M5 brane $\left(\mathrm{AdS}_{7} \otimes S^{4}\right)$ are recovered.

Generalizing the construction to higher levels of exceptional periodicity (parametrized by $n \in \mathbb{N}$ ), where Jordan algebras of degree 3 are lifted to the special class of rank-3 Vinberg's T-algebras [17], maximal embeddings that respect the symmetry of the T-algebraic spin factors yield the spaces $\mathrm{AdS}_{4} \otimes S^{8 n}$ and $\mathrm{AdS}_{8 n-1} \otimes S^{5}$, with reduced subspaces $\mathrm{AdS}_{4} \otimes S^{8 n-1}$ and $\mathrm{AdS}_{8 n-1} \otimes S^{4}$, respectively. Through exceptional periodicity, this suggests generalizations of the M2 brane and M5 brane near-horizon geometries from SYM's in $(8 n+3)+3$ space-time dimensions, as descending from SYM's in $(8 n+4)+4$ space-time dimensions, resulting in an $\mathrm{M}(8 n-3)$ brane dual to the M2 brane [18].

The plan of the paper is as follows.

Within EP, we explicitly study levels $n=1$ (the trivial level, corresponding to exceptional Lie algebras, in particular, to $\mathfrak{e}_{8(-24)}$ in our case), $n=2$ (the first nontrivial level), and $n=3$, respectively, in Secs. II, III B, and III D. Interestingly, at level $n=3$, the resulting branchings give $\mathrm{AdS}_{4} \otimes S^{23}$ and hint at M2 and M21 branes as solutions of the (conjectured) bosonic M-theory [19]; by reduction to $\mathrm{AdS}_{3} \otimes S^{23}$, we recover a space that lends support for Witten's monstrous AdS/CFT construction for threedimensional gravity [20], as the Conway group $\mathrm{Co}_{0}$ (the symmetry of the Leech lattice [21]) is recovered from the $S O(24) \mathcal{R}$-symmetry ${ }^{1}$ of a discretized $S^{23}$. On the other hand, our analysis at levels $n=2$ and 3 can be bridged by the observation that every $\mathrm{K} 3$ sigma model contains a symmetry group that is a subgroup of $\mathrm{Co}_{0}$ [22].

Section III A therein presents evidence for the existence of higher dimensional $\mathcal{N}=(1,0)$ SYM's with $1,2,3$ or 4 timelike dimensions, named exceptional SYM's, stemming from similar constructions given by Bars [4], Sezgin [5,23], and Nishino [6].

\footnotetext{
${ }^{1}$ For $D=27$ M-theory reduced to $D=3$, there is no light cone little group and the expected $\mathcal{R}$-symmetry is the full $S O(24)$, the transversal rotation group in $D=26$. This is the higher dimensional analog of what occurs for $S O(8) \mathcal{R}$-symmetry in $D=11$ M-theory reduced to $D=3$.
}

Such higher dimensional SYM's can be defined at every level of EP for $\mathfrak{e}_{8(-24)}$, whose generic $n$th level is considered in Sec. III C. The resulting "EP/SYM correspondence" (which is investigated further on) suggests a spectral formulation of M-theory from the class of Vinberg's special cubic T-algebras [17]. This generalizes the structure of matrix theory [24] in $D=10+1$ to a more general class of nonassociative matrix operator algebras that periodically exhibit nonassociative geometry, up to infinite dimensions. This is as Connes [25] has done for noncommutative geometry from noncommutative $C^{*}$-algebras, which falls under the more general motivic program of Grothendieck [26].

$$
\text { II. } e_{8}(-24)
$$

\section{A. $\mathcal{N}=(1,0)$ SYM in $11+3$ dimensions}

The Cayley plane $\mathbb{O P}^{2}$ is the projective space of all rank-1 projectors of the exceptional Jordan algebra $\mathbf{J}_{3}^{\mathbb{Q}}$; by stabilizing a point of the Cayley plane, the affine symmetry $E_{6(-26)}$ is reduced to $S O(9,1)[15]$. This $S O(9,1)$ subgroup acts on a line (an $S^{8}$ ) of the Cayley plane as affine transformations. It is also the symmetry of the 10-dimensional spin factor from the Peirce decomposition (cf., e.g., [27,28]),

$$
\mathbf{J}_{3}^{\mathbb{Q}}=\mathbf{1 0} \oplus \mathbf{1 6} \oplus \mathbf{1},
$$

where the fixed point (primitive idempotent of $\mathbf{J}_{3}^{\mathbb{Q}}$ ) corresponds to the $\mathbf{1}$ in the rhs of (2.1), and it serves as a point at infinity for the eight-dimensional transverse space. The affine symmetry and MW semispinor $\mathbf{1 6}$ occur in the 3 -grading ${ }^{2}$ of $\mathfrak{e}_{6(-26)}$,

$$
\mathfrak{e}_{6(-26)}=\mathbf{1 6}_{-3}^{\prime} \oplus\left(\mathfrak{s o}_{9,1} \oplus \mathbb{R}\right)_{0} \oplus \mathbf{1 6}_{+3},
$$

where $16^{\prime}$ is the conjugate MW semispinor in $9+1$ spacetime dimensions. (2.2) can be interpreted as the maximal symmetric embedding

$$
\mathfrak{s t x _ { 0 }}\left(\mathbf{J}_{3}^{\mathbb{Q}}\right) \supset \mathfrak{s t r} \mathfrak{x}_{0}\left(\mathbb{R} \oplus \mathbf{J}_{2}^{\mathbb{Q}}\right),
$$

which is a consequence of the maximal Jordan-algebraic embedding,

$$
\mathbf{J}_{3}^{\mathbb{Q}} \supset \mathbb{R} \oplus \mathbf{J}_{2}^{\mathbb{Q}},
$$

where $\mathbf{J}_{2}^{\mathbb{Q}} \simeq \boldsymbol{\Gamma}_{9,1}$ [cf., e.g., (5.2) of [30]] is a 10-dimensional Lorentzian spin factor $[31,32]$ and $\mathbb{R}$ is the span of the fixed idempotent. ${ }^{3}$

The magic star of $\mathfrak{e}_{8(-24)}$ (cf. Fig. 1 and its caption) is a projection along an $\mathfrak{s l}_{3, \mathbb{R}}$ subalgebra, with $\mathfrak{e}_{6(-26)}$ projected to a central vertex [10-13]. In $\mathfrak{e}_{8(-24)}$, the analog of $\mathfrak{s}_{9,1}$ is $\mathfrak{S}_{12,4}$; indeed, the following maximal symmetric embedding holds:

\footnotetext{
${ }^{2}$ This is a remarkable 3 -grading structure, related to a Jordan pair that is not a pair of Jordan algebras, but rather a Jordan triple system; cf., e.g., [29].

${ }^{3}$ The $\mathbb{R}$ idempotent direction transforms spatially under $\mathfrak{S}_{10,1} \subset$ $\mathfrak{S}_{10,2} \subset \mathfrak{e}_{7(-25)}$, and can serve as an 11th dimension of M-theory.
} 


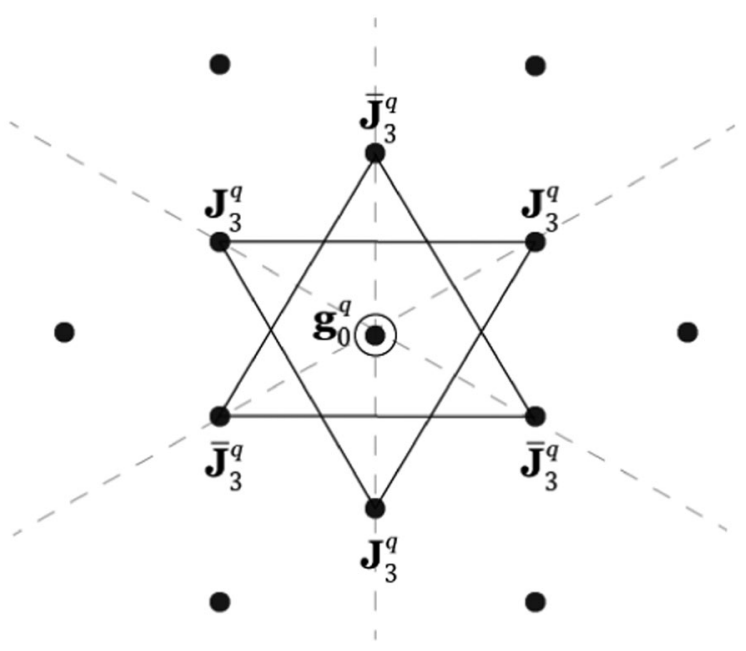

FIG. 1. The magic star of finite-dimensional exceptional Lie algebras [10]. $\mathbf{J}_{3}^{q}$ denotes the Jordan algebra of $3 \times 3$ Hermitian matrices over the division algebras $\mathbb{A}=\mathbb{R}, \mathbb{C}, \mathbb{M}$ and $\mathbb{O}$ for $q=\operatorname{dim}_{\mathbb{R}} \mathbb{A}=1,2,4,8$ respectively, whereas $\mathbf{g}_{\mathbf{0}}^{q}$ denotes its reduced structure Lie algebra. In the case of the minimally noncompact, real form $\mathfrak{e}_{8(-24)}$ of $\mathfrak{e}_{8}$ under consideration, $q=8$ and $\mathbf{g}_{0}^{8}=\mathfrak{e}_{6(-26)}$. In the text, we use $\mathbf{J}_{3}^{\mathbb{Q}} \equiv \mathbf{J}_{3}^{8}$.

$$
\mathfrak{e}_{8(-24)} \supset \mathfrak{S}_{12,4},
$$

interpreted as

$$
\mathfrak{q c o n} \mathfrak{f}\left(\mathbf{J}_{3}^{\mathbb{Q}}\right) \supset \mathfrak{q c o n} \mathfrak{f}\left(\mathbb{R} \oplus \mathbf{J}_{2}^{\mathbb{Q}}\right),
$$

which is still a consequence of (2.4); qconf here denotes the quasiconformal symmetry of the corresponding rank-3 Jordan algebra [33]. The branching corresponding to (2.5) gives naturally rise to the following 5-grading,

$$
\begin{aligned}
\mathfrak{e}_{8(-24)}= & \mathfrak{S o}_{12,4} \oplus \mathbf{1 2 8}=\mathbf{1 4}_{-2} \oplus \mathbf{6 4}_{-1}^{\prime} \oplus\left(\mathfrak{S \mathfrak { p } _ { 1 1 , 3 }} \oplus \mathbb{R}\right)_{0} \\
& \oplus \mathbf{6 4}_{+1} \oplus \mathbf{1 4} \mathbf{4}_{+2},
\end{aligned}
$$

where 64 and $64^{\prime}$ are the MW semispinor and its conjugate in $D=s+t=11+3$ space-time dimensions. Part of (2.7) has already appeared in $[7,8]$. As mentioned above, mathematically, it identifies (the minimally noncompact, real form of) a Kantor triple system, of "extended Poincaré type," over $\mathfrak{e}_{8}$ [9]. Moreover, we recall that $\mathfrak{S}_{11,3}$, occurring in the 0 -graded reductive component of the 5-grading (2.7), is the space-time, purely bosonic, symmetry Lie algebra of the $\mathcal{N}=(1,0)$ SYM studied by Sezgin, Bars, and Nishino in $D=11+3$ space-time dimensions [4-6] (see also Sec. III A below).

\section{B. M2-brane in 10+1 dimensions}

At the Lie group level, a maximal symmetric subgroup of $S O(12,4)$ is

$$
S O(12,4) \supset S O(3,3) \times S O(9,1) .
$$

Recalling the coset

$$
\mathrm{AdS}_{4}=O(3,2) / O(3,1)
$$

$S O(3,2)$ acts on $\mathrm{AdS}_{4}$ as isometries, while $S O(3,3)$ acts via affine transformations. Hence, $S O(12,4)$ includes maximally (and symmetrically) the affine transformations of $\mathrm{AdS}_{4} \otimes S^{8}$.

Reducing to transversal rotations, the isometry group $S O(9)$ of $S^{8} \simeq \mathbb{O P}^{1}$ (which can be regarded as a line in $\mathbb{O P}^{2}$ ) breaks to $S O(8)$ acting on $S^{7}$. This corresponds to the two-step chain of maximal symmetric embeddings into $\operatorname{SO}(12,4)$,

$$
S O(12,4) \supset S O(11,3) \supset S O(3,3) \times S O(8) \text {; }
$$

breaking down to isometries of $\mathrm{AdS}_{4}$ by further maximal and symmetric embedding yields

$$
\begin{aligned}
S O(12,4) \supset & S O(11,3) \supset S O(3,3) \times S O(8) \supset S O(3,2) \\
& \times S O(8) .
\end{aligned}
$$

Alternatively, the following embedding also holds:

$$
S O(12,4) \supset S O(3,2) \times S O(8) \times S O(1,2),
$$

where $S O(3,2) \times S O(8)$ acts as isometries of $\mathrm{AdS}_{4} \otimes S^{7}$, the near-horizon geometry of the M2-brane [whose world volume symmetry is described by $S O(1,2)$ ], as a solution of $10+1$ M-theory (or of its low-energy limit, $\mathcal{N}=1$ eleven-dimensional supergravity); see, e.g., [34-36].

\section{M5 brane in $10+1$ dimensions}

Another maximal symmetric subgroup of $S O(12,4)$ is

$$
S O(12,4) \supset S O(6,3) \times S O(6,1) .
$$

Recalling the coset

$$
\mathrm{AdS}_{7}=O(6,2) / O(6,1)
$$

$S O(6,2)$ acts on $\mathrm{AdS}_{7}$ as isometries, and $S O(6,3)$ acts via affine transformations. Hence, $S O(12,4)$ includes maximally (and symmetrically) the affine transformations of $\mathrm{AdS}_{7} \otimes S^{5}$.

Considering the reduction $S^{5} \rightarrow S^{4}$, where $S^{4} \simeq \mathbb{M} \mathbb{P}^{1}$ can be conceived as a line in $\mathbb{P P}^{2}$, the isometry group $S O(6)$ of $S^{5}$ breaks to $S O(5)$ on $S^{4}$. Thus, the reduction $\mathbb{O} \rightarrow \mathbb{M}$ reduces $\mathbb{O P}^{1} \simeq S^{8} \rightarrow \mathbb{M P} \mathbb{P}^{1} \simeq S^{4}$ with the point at infinity given by a fixed primitive idempotent of $\mathbf{J}_{3}^{\mathbb{N}}$. This corresponds to the following chain of maximal symmetric embeddings into $S O(12,4)$ :

$$
S O(12,4) \supset S O(11,3) \supset S O(6,3) \times S O(5) \text {; }
$$

breaking down to isometries of $\mathrm{AdS}_{7}$ by further maximal and symmetric embedding yields

$$
\begin{aligned}
S O(12,4) & \supset S O(11,3) \supset S O(6,3) \times S O(5) \\
& \supset S O(6,2) \times S O(5) .
\end{aligned}
$$

Alternatively, the following embedding also holds:

$$
S O(12,4) \supset S O(6,2) \times S O(5) \times S O(1,2),
$$


where $S O(6,2) \times S O(5)$ acts as isometries of $\mathrm{AdS}_{7} \otimes S^{4}$, the near-horizon geometry of the M5 brane, Hodge dual to the M2 brane [whose world volume symmetry $S O(1,2)$ still occurs as a commuting factor] in $10+1$ space-time dimensions (see, e.g., [34-36]).

Note also that $S O(12,4)$ contains the isometries of $\mathrm{AdS}_{7}$ and of the M5 brane world volume, times a dilatational factor,

$$
\begin{aligned}
S O(12,4) & \supset S O(6,2) \times S O(5,1) \times S O(1,1) \\
& \simeq S O^{*}(8) \times S U^{*}(4) \times S O(1,1),
\end{aligned}
$$

or, equivalently, through two different maximal and symmetric embeddings, the isometries of $\mathrm{AdS}_{7}$ times the conformal symmetry of the M5 brane world volume, or, respectively, the conformal symmetry of $\mathrm{AdS}_{7}$ times the isometries of the M5 brane world volume,

$S O(12,4) \supset S O(6,2) \times S O(6,2) \simeq S O^{*}(8) \times S O^{*}(8)$;

$S O(12,4) \supset S O(7,3) \times S O(5,1) \simeq S O(7,3) \times S U^{*}(4)$.

\section{GENERALIZATIONS WITHIN EXCEPTIONAL PERIODICITY}

\section{A. Exceptional SYM's}

As mentioned in the introduction, various efforts have been made over the years to define consistent supersymmetric theories in $D>11$ space-time dimensions. For instance, Nishino constructed an $\mathcal{N}=(1,0)$ SYM [37] as well as an $\mathcal{N}=1$ [38] and $\mathcal{N}=2$ [39] supergravity theory in $10+2$ dimensions, with the two timelike dimensions being motivated by the development of F-theory [2,40,41], Bars' S-theory [3], or other two-times theories [42]. Nonminimal, chiral, $\mathcal{N}=(2,0) \mathrm{SYM}$ in $10+2$ was considered by Sezgin (cf., e.g., [23]). More recently, Castellani proposed an $\mathcal{N}=1$ supergravity in $10+2$ with locally supersymmetric $S O(10,2)$-invariant action [43]. Switching to three timelike dimensions, Bars introduced $11+3$ supersymmetry [4] and Sezgin constructed an $\mathcal{N}=$ $(1,0)$ SYM in $11+3$ [5], obtaining a space-time superalgebra with 64 real supercharges containing the type IIB Poincaré superalgebra in $9+1$ as well as $\mathcal{N}=1$ Poincaré superalgebra in $10+1$. However, Sezgin also found an obstruction for extending global supersymmetry beyond $11+3$, later overcome by Nishino [6], who formulated $\mathcal{N}=(1,0)$ SYM's in any signature $(9+m)+(1+m)$, for arbitrary $m \in \mathbb{N} \cup\{0\}$, with four general classes of SYM's given by the fact that $m=4 \mathbf{n}, \mathbf{n} \in \mathbb{N} \cup\{0\}$.

Essentially, Bars [4], Sezgin [5], and then Nishino [6] considered the symmetries of the $\gamma$-matrices [44], in particular, signatures $D=s+t$, all with $s-t=8$. Disregarding the Euclidean case $(t=0)$, four $\mathbf{n}$-parametrized classes of chiral, minimal $\mathcal{N}=(1,0)$ superalgebras can be consistently defined [5,6],

(1) $D=(9+4 \mathbf{n})+(1+4 \mathbf{n})$ :

$$
\begin{aligned}
\left\{Q_{\alpha}, Q_{\beta}\right\}= & \left(\gamma^{\mu}\right)_{\alpha \beta} P_{\mu}+\left(\gamma^{\mu_{1} \ldots \mu_{5}}\right)_{\alpha \beta} Z_{\mu_{1} \ldots \mu_{5}} \\
& +\cdots+\left(\gamma^{\mu_{1} \ldots \mu_{5+4 n}}\right)_{\alpha \beta} Z_{\mu_{1} \ldots \mu_{5+4 n}} .
\end{aligned}
$$

(2) $D=(10+4 \mathbf{n})+(2+4 \mathbf{n})$ :

$$
\begin{aligned}
\left\{Q_{\alpha}, Q_{\beta}\right\}= & \left(\gamma^{\mu \nu}\right)_{\alpha \beta} Z_{\mu \nu}+\left(\gamma^{\mu_{1} \ldots \mu_{6}}\right)_{\alpha \beta} Z_{\mu_{1} \ldots \mu_{6}} \\
& +\cdots+\left(\gamma^{\mu_{1} \ldots \mu_{6+4 n}}\right)_{\alpha \beta} Z_{\mu_{1} \ldots \mu_{6+4 n}} .
\end{aligned}
$$

(3) $D=(11+4 \mathbf{n})+(3+4 \mathbf{n})$ :

$$
\begin{aligned}
\left\{Q_{\alpha}, Q_{\beta}\right\}= & \left(\gamma^{\mu \nu \rho}\right)_{\alpha \beta} Z_{\mu \nu \rho}+\left(\gamma^{\mu_{1} \ldots \mu_{7}}\right)_{\alpha \beta} Z_{\mu_{1} \ldots \mu_{7}} \\
& +\cdots+\left(\gamma^{\mu_{1} \ldots \mu_{7+4 \mathrm{n}}}\right)_{\alpha \beta} Z_{\mu_{1} \ldots \mu_{7+4 \mathrm{n}}} .
\end{aligned}
$$

(4) $D=(12+4 \mathbf{n})+(4+4 \mathbf{n})$ :

$$
\begin{aligned}
\left\{Q_{\alpha}, Q_{\beta}\right\}= & \eta_{\alpha \beta} Z+\left(\gamma^{\mu_{1} \ldots \mu_{4}}\right)_{\alpha \beta} Z_{\mu_{1} \ldots \mu_{4}} \\
& +\left(\gamma^{\mu_{1} \ldots \mu_{8}}\right)_{\alpha \beta} Z_{\mu_{1} \ldots \mu_{8}} \\
& +\cdots+\left(\gamma^{\mu_{1} \ldots \mu_{8+4 n}}\right)_{\alpha \beta} Z_{\mu_{1} \ldots \mu_{8+4 n}} .
\end{aligned}
$$

In such space-time superalgebras, $Z_{\mu_{1} \ldots \mu_{p}}$ are the bosonic p-form generators. Actually, in (3.1)-(3.4) the $\gamma$-matrices are all chirally projected: $\gamma^{\mu_{1} \ldots \mu_{p}} \equiv \gamma^{\mu_{1} \ldots \mu_{p}} C^{-1}$, with $C$ denoting the charge conjugation matrix [the $\eta$-matrix occurring in (3.4) is the chiral projection of $C$ itself]. The maximal rank $\gamma$-matrices in the rhs of (3.1)-(3.4) have a definite duality property, and hence the corresponding bosonic generator is taken to be self-dual. Thus, the rhs of (3.1)-(3.4) span the full symmetric space of relevant dimension, and all the $\gamma$-matrices surviving the chiral projection occur therein. It is here worth pointing out that, among the above chiral superalgebras, only the one in $D=(9+4 \mathbf{n})+(1+4 \mathbf{n})$ space-time dimensions (3.1) is a proper Poincaré superalgebra, containing the momentum operator $P_{\mu}$ in the rhs.

We should also recall that the symmetry property of the $\gamma$-matrices repeats itself every eight dimensions in space-time [44], while the chirality (dottedness) of the spinors alternates every two dimensions. In particular, the properties of spinors are defined by two parameters: $D=s+t \bmod (8)$ and $\rho=s-t \bmod (8)$ [the $\bmod (8)$ periodicity being the Bott periodicity; see, e.g., [45], and references therein]. Thus, the fact that all superalgebras (3.1)-(3.4) are characterized by a MW (semi)spinor generator can be traced back to that they all have $\rho=8=0 \bmod (8)$.

The simple but crucial observation is that, by switching $4 \mathbf{n}$ timelike dimensions into $4 \mathbf{n}$ spacelike dimensions in the above space-time signatures, one keeps $D$ and $\rho$ unchanged, and so all the properties of spinors and $\gamma$-matrices relevant for the definition of the above super-Poincare algebras are left unchanged. Thus, one can check that the proofs given in [6] (see also [5]) for the existence of SYM's with superalgebras 
(3.1) and (3.2) can be successfully repeated to show the existence of SYM's based on global minimal, chiral $(1,0)$ superalgebras in the following space-time signatures:

(i) $D=(9+8 \mathbf{n})+1$ :

$$
\begin{aligned}
\left\{Q_{\alpha}, Q_{\beta}\right\}= & \left(\gamma^{\mu}\right)_{\alpha \beta} P_{\mu}+\left(\gamma^{\mu_{1} \ldots \mu_{5}}\right)_{\alpha \beta} Z_{\mu_{1} \ldots \mu_{5}} \\
& +\cdots+\left(\gamma^{\mu_{1} \ldots \mu_{5+4 n}}\right)_{\alpha \beta} Z_{\mu_{1} \ldots \mu_{5+4 n}} .
\end{aligned}
$$

(2) $D=(10+8 \mathbf{n})+2$ :

$$
\begin{aligned}
\left\{Q_{\alpha}, Q_{\beta}\right\}= & \left(\gamma^{\mu \nu}\right)_{\alpha \beta} Z_{\mu \nu}+\left(\gamma^{\mu_{1} \ldots \mu_{6}}\right)_{\alpha \beta} Z_{\mu_{1} \ldots \mu_{6}} \\
& +\cdots+\left(\gamma^{\mu_{1} \ldots \mu_{6+4 n} \mathrm{n}}\right)_{\alpha \beta} Z_{\mu_{1} \ldots \mu_{6+4 n}} .
\end{aligned}
$$

(3) $D=(11+8 \mathbf{n})+3$ :

$$
\begin{aligned}
\left\{Q_{\alpha}, Q_{\beta}\right\}= & \left(\gamma^{\mu \nu \rho}\right)_{\alpha \beta} Z_{\mu \nu \rho}+\left(\gamma^{\mu_{1} \ldots \mu_{7}}\right)_{\alpha \beta} Z_{\mu_{1} \ldots \mu_{7}} \\
& +\cdots+\left(\gamma^{\mu_{1} \ldots \mu_{7+4 n}}\right)_{\alpha \beta} Z_{\mu_{1} \ldots \mu_{7+4 n}} .
\end{aligned}
$$

(4) $D=(12+8 \mathbf{n})+4$ :

$$
\begin{aligned}
\left\{Q_{\alpha}, Q_{\beta}\right\}= & \eta_{\alpha \beta} Z+\left(\gamma^{\mu_{1} \ldots \mu_{4}}\right)_{\alpha \beta} Z_{\mu_{1} \ldots \mu_{4}} \\
& +\left(\gamma^{\mu_{1} \ldots \mu_{8}}\right)_{\alpha \beta} Z_{\mu_{1} \ldots \mu_{8}}+\cdots \\
& +\left(\gamma^{\mu_{1} \ldots \mu_{8+4 n}}\right)_{\alpha \beta} Z_{\mu_{1} \ldots \mu_{8+4 n}} .
\end{aligned}
$$

The SYM's whose space-time (super-Poincaré) superalgebra is given by (3.5)-(3.8) are named exceptional SYM's henceforth. Note that $\mathbf{n}=n-1$, where $n \in \mathbb{N}$ is the level of EP $[13,14,46]$. Thus, a direct relation between EP and higher dimensional, exceptional $(1,0)$ SYM's in $D=(9+8 n)+1$, $D=(10+8 n)+2, D=(11+8 n)+3$, and $D=(12+8 n)+4$ exists, ${ }^{4}$ hinting to the existence of an EP/SYM

\footnotetext{
${ }^{4}$ It should be pointed out that there exists an intrinsic, threefold degeneracy in the determination of the noncompact real forms of EP algebras. Indeed, at the $n$th level of EP, in the present paper (as it is evident from Sec. III C) we understand to enlarge by $8 n$ only the spacelike dimensions in the $(s+t)$-signature of the reductive, pseudo-orthogonal part of the aforementioned EP algebras, thus obtaining $(9+8 n)+1,(10+8 n)+2,(11+8 n)+3$, and $(12+8 n)+4$, and matching (by considering that $\mathbf{n}=n-1$ ) the space-time signatures (3.5)-(3.8). Nevertheless, the conjugation and reality properties of spinors depend only on $D=s+t$ and on $\rho:=s-t$ (cf., e.g., [45]); thus at the $n$th level of EP, the implementation of Bott [i.e., $\bmod (8 n)]$ periodicity could also be made by increasing by $4 n$ both spacelike and timelike dimensions, or also by increasing by $8 n$ only the timelike dimensions. In the former case one would obtain $(9+4 n)+(1+4 n),(10+4 n)+$ $(2+4 n),(11+4 n)+(3+4 n)$, and $(12+4 n)+(4+4 n)$, thus giving rise (by considering that $\mathbf{n}=n-1$ ) to the space-time signatures of (3.1)-(3.4), whereas in the latter case, one would obtain 9+ $(1+8 n), 10+(2+8 n), 11+(3+8 n)$, and $12+(4+8 n)$, thus giving rise (by considering that $\mathbf{n}=n-1$ ) to other space-time signatures in which other chiral, minimal $\mathcal{N}=(1,0)$ superalgebras, besides (3.1)-(3.4) and (3.5)-(3.8), exist. Such a threefold degeneracy of the implementation of Bott periodicity (yielding spinors with the same dimensions, reality properties, and conjugation properties) can in principle be applied at any level of EP, also in a different way from the way it was implemented at the previous level; this allows one to span a large variety of $(s, t)$-signatures in the $\mathfrak{S}_{\mathfrak{o}_{s, t}}$ reductive part of the noncompact real forms of EP algebras [18].
}

correspondence [18]. In particular, the levels $n=1,2,3$ (and for a generic $n$ ) of the class of SYM's in $D=$ $(11+8 n)+3$ space-time dimensions with superalgebra (3.7) are considered in the present paper.

\section{B. The first nontrivial level $(n=2): e_{8(-24)}^{(2)}$}

\section{1. $\mathcal{N}=(1,0) S Y M$ in $19+3$ dimensions}

At the second level $(n=2)$ of EP [13], one can define

$$
\mathfrak{e}_{8(-24)}^{(2)}:=\mathfrak{S}_{20,4} \oplus \mathbf{2 0 4 8},
$$

with algebraic structure and commutation relations defined in terms of Kac's asymmetry function [13], and with 2048 denoting the MW semispinor in $20+4$ space-time dimensions. (3.9) is the very first step of a Bott-periodic, non-Lie generalization $^{5}$ of $\mathfrak{e}_{8(-24)} \equiv \mathfrak{e}_{8(-24)}^{(1)}$, and it gives naturally rise to the following 5-grading:

$\mathfrak{e}_{8(-24)}^{(2)}=\mathbf{2 2}_{-2} \oplus \mathbf{1 0 2 4}_{-1}^{\prime} \oplus\left(\mathfrak{S}_{19,3} \oplus \mathbb{R}\right)_{0} \oplus \mathbf{1 0 2 4}_{+1} \oplus \mathbf{2 2}_{+2}$,

where $\mathbf{1 0 2 4}$ and $\mathbf{1 0 2 4}^{\prime}$ are the MW semispinor and its conjugate in $19+3$ space-time dimensions.

Equation (3.10) is the first nontrivial generalization of the 5-grading (2.7) of $\mathfrak{e}_{8(-24)}$, and, in light of the discussion in Sec. III A, it provides the vector and spinor structures for a novel, exceptional $\mathcal{N}=(1,0) \mathrm{SYM}$ in $19+3$, generalizing [18] the work of Bars, Sezgin, and Nishino [4-6,23]. It is worth stressing that $19+3$ is the signature of a unimodular lattice appearing in the description of the space of periods for a complex $K 3$ surface $S$ and a Kahler class of $H^{1,1}(S, \mathbb{R})$ [47].

Also, the moduli space of $\mathcal{N}=(4,4)$ string theories with $\mathrm{K} 3$ target space has a discrete symmetry group that is the integral orthogonal group of an even unimodular lattice of signature $(20,4)$ [48]. This may permit further application of (3.10) (with normalized cocycle) in the study of vertex operator algebras for BPS states of K3 sigma models with Mathieu group $\mathbb{M}_{24}$ symmetry [22].

\section{M2 brane in $18+1$ dimensions}

Considering the Lie group associated to the reductive (simple) part of $\mathrm{e}_{8(-24)}^{(2)}$, namely $S O(20,4)$, we observe that a maximal symmetric subgroup of this reads

$$
S O(20,4) \supset S O(3,3) \times S O(17,1) .
$$

Again, $S O(3,3)$ yields affine transformations of $\mathrm{AdS}_{4}$. On the other hand, $S O(17,1)$ can be regarded as the affine symmetry of $S^{16}$, which is the sphere acquired from $\mathbf{T}_{3}^{8,2}$, the rank-3 T-algebra of special type [17], which provides the first nontrivial generalization of the Albert algebra $\mathbf{J}_{3}^{\mathbb{Q}} \equiv$ $\mathbf{J}_{3}^{8} \equiv T_{3}^{8,1}$ within EP (cf. Fig. 2). Fixing a rank-1 idempotent

\footnotetext{
${ }^{5}$ This algebra was inspired by discussions with Eric Weinstein on extended triality in $D=24$.
} 
of $\mathbf{T}_{3}^{8,2}$ induces the following $S O(17,1)$-covariant Peirce decomposition [13]:

$$
\mathbf{T}_{3}^{2}=18 \oplus \mathbf{2 5 6} \oplus \mathbf{1},
$$

where 256 denotes the MW semispinor in signature $17+1$, and 1 is the fixed rank-1 idempotent of $\mathbf{T}_{3}^{8,2}$. (3.12) can be regarded as a consequence of the maximal embedding $[13,46]$

$$
T_{3}^{2} \supset \mathbb{R} \oplus \Gamma_{17,1},
$$

which in turn might give rise to a quasiconformal interpretation of the definition (3.9) itself [14]. The 18-dimensional Lorentzian spin factor $\boldsymbol{\Gamma}_{17,1}[49]$ has $S O(17,1)$ space-time symmetry, which is also the affine symmetry of $S^{16}$, a sphere of the transverse degrees of freedom with a fixed (rank-1 idempotent) point at infinity. It is here worth recalling that this structure is seen in the 3-grading of the first nontrivial extension $\mathfrak{e}_{6(-26)}^{(2)}$ of $\mathfrak{e}_{6(-26)}$ within EP [46],

$$
\mathfrak{e}_{6(-26)}^{(2)}=\mathbf{2 5 6}_{-3}^{\prime} \oplus\left(\mathfrak{s o}_{17,1} \oplus \mathbb{R}\right)_{0} \oplus \mathbf{2 5 6}_{+3},
$$

which might enjoy a reduced structure symmetry interpretation, as well [14]. Hence, $S O(20,4)$ contains maximally (and symmetrically) the affine symmetries of $\mathrm{AdS}_{4} \otimes S^{16}$.

Considering the reduction to transversal rotations ${ }^{6}$ $S^{16} \rightarrow S^{15}$, and the isometry group $S O(17)$ reduces to $S O(16)$. This corresponds to the two-step chain of maximal symmetric embeddings into $S O(20,4)$,

$$
S O(20,4) \supset S O(19,3) \supset S O(3,3) \times S O(16) .
$$

Breaking down to isometries of $\mathrm{AdS}_{4}$ by a further maximal and symmetric embedding yields

$$
\begin{aligned}
S O(20,4) \supset & S O(19,3) \supset S O(3,3) \times S O(16) \supset S O(3,2) \\
& \times S O(16) .
\end{aligned}
$$

Alternatively, the following embedding also holds:

$$
S O(20,4) \supset S O(3,2) \times S O(16) \times S O(1,2),
$$

where $S O(3,2) \times S O(16)$ acts as isometries of $\mathrm{AdS}_{4} \otimes S^{15}$, which can thus be regarded as a generalization of the nearhorizon geometry of the M2-brane in $18+1$ space-time dimensions.

\footnotetext{
${ }^{6}$ Note that the geometric picture in terms of projective lines in higher projective spaces is lost for all nontrivial levels of EP, namely for $n \geq 2$, because octonionic (projective) geometry is defined only until dimension 2. This is also reflected in the fact that $\mathbf{T}_{3}^{8,2}$ is not a rank-3 Jordan algebra, and the triality among its block components within $S O(16)$-covariant Peirce decomposition is spoiled [13]. Again, another consequence of the aforementioned fact is that $E P$ algebras are not Lie algebras (because the Jacobi identity does not hold on their nonreductive component [13]). However, after Rosenfeld [50], the isometry $S O(16)$ of $S^{15}$ can be regarded as the stabilizer of $(\mathbb{O} \otimes \mathbb{O}) \mathbb{P}^{2}=E_{8} / S O(16)$, which is an example (with the largest exceptional global isometry) of the so-called Tits' buildings [51,52].
}

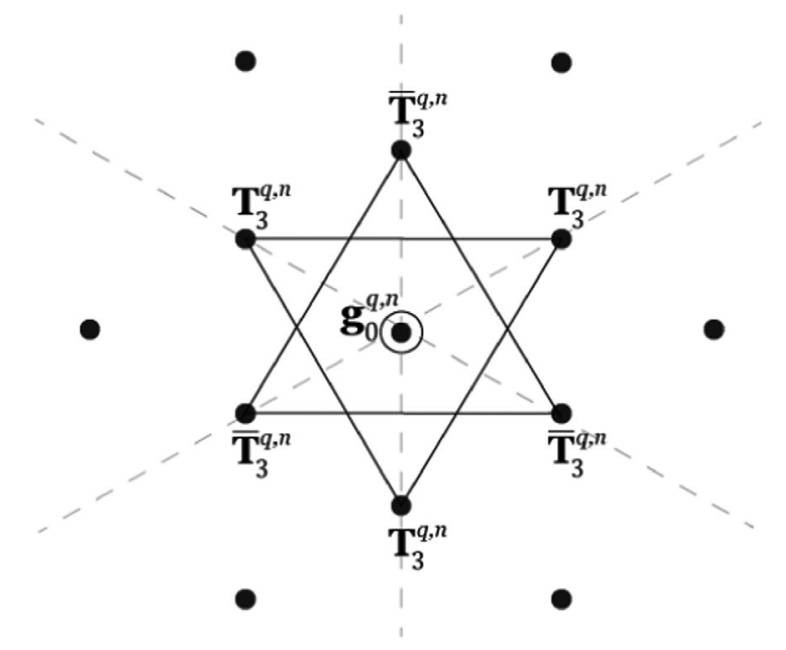

FIG. 2. The magic star of EP [13]. $\mathbf{T}_{3}^{q, n}$ denotes a T-algebra of rank-3 and of special type [17], parametrized by $q=\operatorname{dim}_{\mathbb{R}} \mathbb{A}=$ $1,2,4,8$ for $\mathbb{A}=\mathbb{R}, \mathbb{C}, \mathbb{H}, \mathbb{O}$, respectively, and $n \in \mathbb{N}[17,46]$. In the case of the EP for $\mathfrak{e}_{8(-24)}$, as under discussion, $q=8$.

\section{M13-brane in $18+1$ dimensions}

Another maximal symmetric subgroup of $S O(20,4)$ is

$$
S O(20,4) \supset S O(14,3) \times S O(6,1)
$$

yielding that $S O(20,4)$ includes maximally (and symmetrically) the affine transformations of $\mathrm{AdS}_{15} \otimes S^{5}$. Restricting to transverse directions induces $S^{5} \rightarrow S^{4}$, where the isometry group $S O(6)$ of $S^{5}$ breaks to $S O(5)$ of $S^{4}$. This corresponds to the two-step chain of maximal symmetric embeddings $S O(12,4)$,

$$
S O(20,4) \supset S O(19,3) \supset S O(14,3) \times S O(5)
$$

Breaking down to isometries of $\mathrm{AdS}_{15}$ by a further maximal and symmetric embedding yields

$$
\begin{aligned}
S O(20,4) \supset & S O(19,3) \supset S O(14,3) \times S O(5) \supset S O(14,2) \\
& \times S O(5) .
\end{aligned}
$$

Alternatively, the following embedding also holds:

$$
S O(20,4) \supset S O(14,2) \times S O(5) \times S O(1,2),
$$

where $S O(14,2) \times S O(5)$ acts as isometries of $\mathrm{AdS}_{15} \otimes S^{4}$, which can thus be regarded as a generalization of the nearhorizon geometry $\mathrm{AdS}_{7} \otimes S^{4}$ [cf. (2.17)] of the M5 brane in $10+1$ to the near-horizon geometry of an M13-brane, which is the Hodge dual ${ }^{7}$ of M2 in $18+1$ space-time dimensions.

\footnotetext{
${ }^{7}$ An M13 brane is indeed recovered by taking the Hodge dual of the 4-form field strength of the M2 in $D=18+1$ space-time, sourcing a 15-form dual field strength.
} 


\section{The generic $n$th level $(n \in \mathbb{N}): e_{8(-24)}^{(n)}$}

\section{Infinite $\mathcal{N}=(1,0)$ SYM's in $(8 n+3)+3$ dimensions}

The procedure of the previous subsection can be generalized to an arbitrary level $n$ of EP (characterized by Bott periodicity) as follows [13] $\left(n \in \mathbb{N}\right.$; recall that $\mathfrak{e}_{8(-24)} \equiv$ $\left.\mathfrak{e}_{8(-24)}^{(1)}\right)$ :

$$
\begin{aligned}
\mathfrak{e}_{8(-24)}^{(n)}:= & \mathfrak{S}_{8 n+4,4} \oplus \mathbf{2}^{(8 n+6) / 2} \\
= & (\mathbf{8} \mathbf{n}+\mathbf{6})_{-2} \oplus\left(\mathbf{2}^{(8 n+6) / 2-1}\right)_{-1}^{\prime} \\
& \oplus\left(\mathfrak{S o}_{8 n+3,3} \oplus \mathbb{R}\right)_{0} \oplus \mathbf{2}_{-1}^{(8 n+6) / 2-1} \\
& \oplus(\mathbf{8} \mathbf{n}+\mathbf{6})_{+2},
\end{aligned}
$$

where $\mathbf{2}^{(8 n+6) / 2}$ is the MW semispinor in $(8 n+4)+4$ space-time dimensions, while $\mathbf{2}^{(8 n+6) / 2-1}$ and $\left(\mathbf{2}^{(8 n+6) / 2-1}\right)^{\prime}$ denote the MW spinor and its conjugate in $(8 n+3)+3$ space-time dimensions. (3.22) and (3.23) respectively provide the $n$th generalization of (3.9) and (3.10) within EP [13]. Again, in light of the discussion in Sec. III A, this is tantalizing evidence for the possible existence of a countably infinite tower (parametrized by $n \in \mathbb{N}$ ) of novel, exceptional $\mathcal{N}=(1,0)$ SYM's in $(8 n+3)+3$ space-time dimensions. This generalization of the work by Bars et al. [4-6,23], briefly considered in Sec. III A, is the object of a forthcoming paper [18].

\section{M2 brane in $(8 n+2)+1$ dimensions}

Considering the Lie group associated to the reductive (simple) part of $\mathbf{e}_{8(-24)}^{(n)}$, namely, $S O(8 n+4,4)$, we observe that a maximal symmetric subgroup of this reads

$$
S O(8 n+4,4) \supset S O(3,3) \times S O(8 n+1,1) .
$$

Once again, $S O(3,3)$ yields affine transformations of $\mathrm{AdS}_{4}$. On the other hand, $S O(8 n+1,1)$ can be regarded as the affine symmetry of $S^{8 n}$, which is the sphere acquired from $\mathbf{T}_{3}^{8, n}$, the rank-3 T-algebra of special type [17] that provides the $n$th generalization of the Albert algebra $\mathbf{J}_{3}^{\mathbb{Q}} \equiv \mathbf{J}_{3}^{8} \equiv T_{3}^{8,1}$ within EP (cf. Fig. 2). Fixing a rank-1 idempotent of $\mathbf{T}_{3}^{8, n}$ induces the following $S O(8 n+1,1)$-covariant Peirce decomposition [13]:

$$
\mathbf{T}_{3}^{8, n}=(\mathbf{8} \mathbf{n}+\mathbf{2}) \oplus \mathbf{2}^{4 n} \oplus \mathbf{1},
$$

where $\mathbf{2}^{4 n}$ denotes the MW semispinor in signature $(8 n+1)+1$, and 1 is the fixed rank-1 idempotent of $\mathbf{T}_{3}^{8, n}$. (3.25) can be regarded as a consequence of the maximal embedding $[13,46]$

$$
\mathbf{T}_{3}^{8, n} \supset \mathbb{R} \oplus \boldsymbol{\Gamma}_{8 n+1,1},
$$

which in turn might give rise to a quasiconformal interpretation of the definition (3.22) itself [14]. The $(8 n+2)$ dimensional Lorentzian spin factor $\boldsymbol{\Gamma}_{8 n+1,1}$ has $S O(8 n+1,1)$ space-time symmetry, which is also the affine symmetry of $S^{8 n}$, a sphere of the transverse directions with a fixed idempotent point at infinity. It is here worth recalling that this structure is seen in the 3 -grading of the $n$th generalization $\mathfrak{e}_{6(-26)}^{(n)}$ of $\mathfrak{e}_{6(-26)}$ within EP [46],

$$
\mathfrak{e}_{6(-26)}^{(n)}=\left(\mathbf{2}^{4 n}\right)_{-3}^{\prime} \oplus\left(\mathfrak{s}_{8 n+1,1} \oplus \mathbb{R}\right)_{0} \oplus \mathbf{2}_{+3}^{4 n},
$$

which might enjoy a reduced structure symmetry interpretation, as well [14]. Hence, $S O(8 n+4,4)$ contains maximally (and symmetrically) the affine symmetries of $\mathrm{AdS}_{4} \otimes S^{8 n}$.

Considering the reduction to transversal rotations ${ }^{8}$ $S^{8 n} \rightarrow S^{8 n-1}$, and the isometry group $S O(8 n+1)$ reduces to $S O(8 n)$. This corresponds to the two-step chain of maximal symmetric embeddings into $S O(8 n+4,4)$,

$S O(8 n+4,4) \supset S O(8 n+3,3) \supset S O(3,3) \times S O(8 n)$.

Breaking down to isometries of $\mathrm{AdS}_{4}$ by a further maximal and symmetric embedding yields

$$
\begin{aligned}
S O(8 n+4,4) & \supset S O(8 n+3,3) \supset S O(3,3) S O(8 n) \\
& \supset S O(3,2) \times S O(8 n) .
\end{aligned}
$$

Alternatively, the following embedding also holds:

$S O(8 n+4,4) \supset S O(3,2) \times S O(8 n) \times S O(1,2)$,

where $S O(3,2) \times S O(8 n)$ acts as isometries of $\mathrm{AdS}_{4} \otimes$ $S^{8 n-1}$, which can thus be regarded as a generalization of the near-horizon geometry of the M2 brane in $(8 n+2)+1$ space-time dimensions, i.e., up to arbitrarily high Lorentzian space-times.

\section{3. $M(8 n-3)$ brane in $(8 n+2)+1$ dimensions}

Another maximal symmetric subgroup of $S O(8 n+4,4)$ is

$$
S O(8 n+4,4) \supset S O(8 n-2,3) \times S O(6,1),
$$

yielding that $S O(8 n+4,4)$ maximally (and symmetrically) includes the affine transformations of $\operatorname{AdS}_{8 n-1} \otimes S^{5}$. Considering the reduction to transverse directions induces $S^{5} \rightarrow S^{4}$, where the isometry group $S O(6)$ of $S^{5}$ breaks to $S O(5)$ of $S^{4}$. This corresponds to the two-step chain of maximal symmetric embeddings $S O(8 n+4,4)$,

\footnotetext{
${ }^{8}$ For $n \geq 3$, the interpretation of $S^{8 n}$ (or its reduced space $S^{8 n-1}$ ) in terms of higher projective spaces, or (of stabilizers) of Tits' buildings, is generally lost. For further details, cf. [46].
} 
$S O(8 n+4,4) \supset S O(8 n+3,3) \supset S O(8 n-2,3) \times S O(5)$.

Breaking down to isometries of $\mathrm{AdS}_{7}$ by further maximal and symmetric embedding yields

$$
\begin{aligned}
S O(8 n+4,4) & \supset S O(8 n+3,3) \supset S O(8 n-2,3) \times S O(5) \\
& \supset S O(8 n-2,2) \times S O(5) .
\end{aligned}
$$

Alternatively, the following embedding also holds:

$S O(8 n+4,4) \supset S O(8 n-2,2) \times S O(5) \times S O(1,2)$,

where $S O(8 n-2,2) \times S O(5)$ acts as isometries of $\mathrm{AdS}_{8 n-1} \otimes$ $S^{4}$, which can thus be regarded as a generalization of the nearhorizon geometry of the M5-brane to an $\mathrm{M}(8 n-3)$-brane, which is the Hodge dual of M2. ${ }^{9}$ It is worth noting that the magnetic (Hodge) dual branes of M2-branes can be classified by quaternions as $\mathrm{M}(4 k+1)$ branes: $\mathrm{M} 5$ brane $(k=1), \mathrm{M} 13$ brane ( $k=3)$, M21 brane $(k=5)$, etc. over odd $k=2 n-1$. This provides a generalization of the observation of the projective line in $\mathbb{H} \mathbb{P}^{2}$, done above $(2.15)$, in $(8 n+2)+1$ space-time dimensions, i.e., up to arbitrarily high Lorentzian space-times.

\section{The third level $(n=3): e_{8(-24)}^{(3)}$, bosonic M-theory, and monster AdS/CFT}

\section{1. $\mathcal{N}=(1,0) S Y M$ in $27+3$ dimensions}

As an interesting example, let us consider the generalization of $\mathfrak{e}_{8(-24)}$ provided by the $n=3$ level of EP [13], namely,

$$
\begin{aligned}
\mathfrak{e}_{8(-24)}^{(3)} & :=\mathfrak{S}_{28,4} \oplus \mathbf{2}^{15} \\
& =\mathbf{3 0}_{-2} \oplus\left(\mathbf{2}^{14}\right)_{-1}^{\prime} \oplus\left(\mathfrak{S o}_{27,3} \oplus \mathbb{R}\right)_{0} \oplus \mathbf{2}_{+1}^{14} \oplus \mathbf{3 0}_{+2},
\end{aligned}
$$

where $\mathbf{2}^{15}$ is the MW semispinor in $28+4$, while $2^{14}$ and $\left(\mathbf{2}^{14}\right)^{\prime}$ denote the MW spinor and its conjugate in $27+3$. (3.35) and (3.36), respectively, are the $n=3$ elements of the Bott-periodized countably infinite sequences of generalizations (3.22) and (3.23) within EP. As discussed in Sec. III A, (3.35) and (3.36) intriguingly provide evidence for the possible existence of a novel $\mathcal{N}=(1,0) \mathrm{SYM}$ in $27+3$ [18], generalizing previous works of Bars et al. [4-6,23].

\footnotetext{
${ }^{9} \mathrm{An} \mathrm{M}(8 n-3)$ brane is expected to source the $(8 n-1)$-form field strength, dual to the M2 4-form field strength in $D=$ $(8 n+2)+1$ space-time.
}

\section{M2 brane in bosonic M-theory}

Considering the Lie group associated to the reductive (simple) part of $\boldsymbol{e}_{8(-24)}^{(3)}$, namely, $S O(28,4)$, we observe that a maximal symmetric subgroup of this reads

$$
S O(28,4) \supset S O(3,3) \times S O(25,1),
$$

where $S O(3,3)$ yields affine transformations of $\mathrm{AdS}_{4}$ and $S O(25,1)$ can be regarded as the affine symmetry of $S^{24}$, or alternatively as the space-time symmetry of bosonic string theory [19]. By specializing to $n=3$ the previous $n$-parametrized treatment, $S O(25,1)$ can be regarded as the affine symmetry of $S^{24}$, which is the sphere acquired from $\mathbf{T}_{3}^{8,3}$, the rank-3 T-algebra of special type [17] that provides the third generalization of the Albert algebra within EP (cf. Fig. 2). Fixing a rank-1 idempotent of $\mathbf{T}_{3}^{8,3}$ induces the following $S O(25,1)$-covariant Peirce decomposition [13]:

$$
\mathbf{T}_{3}^{8,3}=\mathbf{2 6} \oplus \mathbf{2}^{12} \oplus \mathbf{1},
$$

where $2^{12}$ denotes the MW semispinor in signature $25+1$, and 1 is the fixed rank-1 idempotent of $\mathbf{T}_{3}^{8,3}$. (3.38) can be regarded as a consequence of the maximal embedding $[13,46]$

$$
\mathbf{T}_{3}^{8,3} \supset \mathbb{R} \oplus \boldsymbol{\Gamma}_{25,1} .
$$

The 26-dimensional Lorentzian spin factor $\boldsymbol{\Gamma}_{25,1}$ has bosonic string theory space-time symmetry, which is also the affine symmetry of $S^{24}$, a sphere of the transverse degrees of freedom with fixed idempotent point at infinity. The span of this fixed idempotent can serve as a 27th dimension for $D=$ 27 M-theory [19]. Note, bosonic string theory space-time symmetry is seen in the 3 -grading of the third generalization $\mathfrak{e}_{6(-26)}^{(3)}$ of $\mathfrak{e}_{6(-26)}$ within EP [46],

$$
\mathfrak{e}_{6(-26)}^{(3)}=\left(\mathbf{2}^{12}\right)_{-3}^{\prime} \oplus\left(\mathfrak{s}_{25,1} \oplus \mathbb{R}\right)_{0} \oplus \mathbf{2}_{+3}^{12} .
$$

Hence, $S O(28,4)$ contains maximally (and symmetrically) the affine symmetries of $\mathrm{AdS}_{4} \otimes S^{24}$.

Considering the reduction to transversal rotations, $S^{24} \rightarrow S^{23}$, and the isometry group $S O(25)$ reduces to $S O(24)$. This corresponds to the two-step chain of maximal symmetric embeddings into $S O(28,4)$,

$$
S O(28,4) \supset S O(27,3) \supset S O(3,3) \times S O(24) .
$$

Breaking down to isometries of $\mathrm{AdS}_{4}$ by a further maximal and symmetric embedding yields

$$
\begin{aligned}
S O(28,4) \supset & S O(27,3) \supset S O(3,3) \times S O(24) \supset S O(3,2) \\
& \times S O(24) .
\end{aligned}
$$


Alternatively, the following embedding also holds:

$$
S O(28,4) \supset S O(3,2) \times S O(24) \times S O(1,2),
$$

where $S O(3,2) \times S O(24)$ acts as isometries of $\mathrm{AdS}_{4} \otimes$ $S^{23}$, which can thus be regarded as a generalization of the near-horizon geometry of the M2 brane in $26+1$ spacetime dimensions. $\mathrm{AdS}_{4} \otimes S^{23}$ might also serve as a possible vacuum of bosonic M-theory [19].

\section{M21 brane in bosonic M-theory}

Another maximal symmetric subgroup of $S O(28,4)$ is

$$
S O(28,4) \supset S O(22,3) \times S O(6,1),
$$

yielding that $S O(28,4)$ maximally (and symmetrically) includes the affine transformations of $\mathrm{AdS}_{23} \otimes S^{5}$. Considering the reduction to transverse directions maps $S^{5} \rightarrow S^{4}$, and the isometry group $S O(6)$ of $S^{5}$ breaks to $S O(5)$ on $S^{4}$. This corresponds to the two-step chain of maximal symmetric embeddings $S O(28,4)$,

$$
S O(28,4) \supset S O(27,3) \supset S O(22,3) \times S O(5) .
$$

Breaking down to isometries of $\mathrm{AdS}_{23}$ by a further maximal and symmetric embedding yields

$$
\begin{aligned}
S O(28,4) \supset & S O(27,3) \supset S O(22,3) \times S O(5) \supset S O(22,2) \\
& \times S O(5) .
\end{aligned}
$$

Alternatively, the following embedding also holds:

$$
S O(28,4) \supset S O(22,2) \times S O(5) \times S O(1,2),
$$

where $S O(22,2) \times S O(5)$ acts as isometries of $\mathrm{AdS}_{23} \otimes$ $S^{4}$, which can thus be regarded as a generalization of the near-horizon geometry of the M5 brane to an M21 brane, the Hodge dual of the M2 brane in $26+1$ space-time dimensions. In view of the considerations above and Horowitz and Susskind's conjectured M21 brane in [19], $\mathrm{AdS}_{23} \otimes S^{4}$ would also provide support for a possible vacuum of bosonic M-theory, "dual" to $\mathrm{AdS}_{4} \otimes S^{23}$.

\section{Conway group and Witten's monster AdS/CFT}

By reducing to isometries of

$$
\mathrm{AdS}_{3}=O(2,2) / O(2,1)
$$

the following embedding into $S O(28,4)$ is singled out:

$$
S O(28,4) \supset S O(2,2) \times S O(24) \times S O(2,2),
$$

where $S O(2,2) \times S O(24)$ yields isometries of $\mathrm{AdS}_{3} \otimes S^{23}$.
We note that the space $\mathrm{AdS}_{3} \otimes S^{23}$ is especially interesting, as it lends support for the monster AdS/ CFT for three-dimensional gravity proposed by Witten [20]. If we suppose $\mathrm{AdS}_{3} \otimes S^{23}$ is a possible vacuum of bosonic string theory, ${ }^{10}$ where the expected $\mathcal{R}$-symmetry is $S O$ (24) (from $D=26+1$ M-theory reduced to $D=2+1)$, we can identify the Conway group $\mathrm{Co}_{0} \subset$ $\mathbb{M}$ (where $\mathbb{M}$ is the monster group) in $S O(24)$ as acting on a discretized $S^{23}$ with points given by vectors of the Leech lattice [21,53], in which the first shell of 196,560 vectors has norm 4 [21]. This allows a finite group action as the Conway group $\mathrm{Co}_{0}$ is the automorphism group of the Leech lattice [21], acting as isometries of the discretized ${ }^{11}$ $S^{23}$. The appearance of the Conway group is more than fortuitous, as every $K 3$ sigma model has finite group symmetry contained in $\mathrm{Co}_{0}$ [22]. This is a tantalizing hint that, beyond the search for vertex operator algebras on BPS states with exact $\mathbb{M}_{24}$ symmetry [22], one can move to a larger vertex operator algebra (CFT) with manifest Conway group symmetry and study its related $K 3$ sigma models. Intriguingly, a super vertex operator algebra with $\mathrm{Co}_{0}$ Conway group symmetry has already been constructed in [54]. An AdS/CFT study of $\mathrm{AdS}_{3} \otimes S^{23}$ might involve this particular vertex operator algebra. In light of this algebra, the monster group could be a finite symmetry of the light cone little group of nonperturbative ${ }^{12} 27-$ dimensional M-theory.

\section{CONCLUSION}

By relying on the maximal embedding (3.26) of semisimple rank-3 Jordan algebras into rank-3 T-algebras of special class, we used 3 - and 5-gradings of finitedimensional exceptional Lie algebras and their Bott-periodic extensions within EP, in order to show that the near-horizon geometries of the M2 branes and their Hodge (magnetic) duals can be generalized to arbitrarily high dimensions.

Moreover, we showed how the EP generalizations of the minimally noncompact, real form $\mathfrak{e}_{8(-24)}$ of the largest finite-dimensional exceptional Lie algebra $\mathfrak{e}_{8}$ hint at novel, exceptional SYM's beyond $11+3$ space-time dimensions, with tantalizing signatures $17+1,19+3,25+1$ and $27+3$, suggesting a periodic ladder to the $D=25+1$ bosonic string (with an $\mathrm{AdS}_{3} \otimes S^{23}$ vacuum) and $D=$ $26+1$ bosonic M-theory (with M2 $\mathrm{AdS}_{4} \otimes S^{23}$ nearhorizon geometry), that can be generalized to arbitrarily high dimension with $1,2,3$ or 4 timelike dimensions.

\footnotetext{
${ }^{10}$ Thanks to Lubos Motl for comments on this construction.

${ }^{11}$ It is tempting to observe that the spherical parts of the corresponding geometries for the levels $n=1$ and $n=2$ of EP, namely, of $\mathrm{AdS}_{3} \otimes S^{7}$ and $\mathrm{AdS}_{3} \otimes S^{15}$, can be discretized by using $E_{8}$ and $E_{8} \oplus E_{8}$ lattices, respectively.

${ }^{12}$ Formally, such a formulation would exist at levels $n=5$ and $n=6$ of EP, where a 24-dimensional even unimodular lattice and its extensions can be constructed.
} 
This allowed us to argue that an EP/SYM correspondence can be put forward [18], implying an infinite-dimensional spectral extension of M-theory via cubic matrix T-algebras, where $D=s+t=10+1$ is the maximal single-time extension of SYM exhibiting manifest $(S O(8))$ triality.

The appearance of $19+3$ and $20+4$ signatures in moduli spaces with $K 3$ target space [47,48], and Mathieu moonshine in $K 3$ sigma models [22], with AdS constructions descending from $28+4$ exhibiting Conway group $\mathrm{Co}_{0}$ symmetry, all yield evidence that higher dimensional SYM's in the EP context (whose existence has been briefly discussed in Sec. III A and will be investigated in detail in [18]) can help shed light on the "mysterious duality" [55] of Iqbal, Nietzke, and Vafa, as well as on the moonshine structure of M-theory and beyond.

Forthcoming companion papers and studies will explore these issues in deeper detail $[14,18,46]$.

\section{ACKNOWLEDGMENTS}

This article presented by M. R. at the symposium symmetries and order: algebraic methods in many Body Systems in honor of Prof. Francesco Iachello, Wright Laboratory and Yale University Physics Department.
[1] E. Witten, String theory dynamics in various dimensions, Nucl. Phys. B443, 85 (1995).

[2] C. Vafa, Evidence for F-theory, Nucl. Phys. B469, 403 (1996).

[3] I. Bars, S-theory, Phys. Rev. D 55, 2373 (1997).

[4] I. Bars, A case for 14 dimensions, Phys. Lett. B 403, 257 (1997).

[5] E. Sezgin, Super Yang-Mills in $(11,3)$ dimensions, Phys. Lett. B 403, 265 (1997).

[6] H. Nishino, Supersymmetric Yang-Mills theories in D $\geq 12$, Nucl. Phys. B523, 450 (1998).

[7] A. G. Lisi, Lie group cosmology, arXiv:1506.08073.

[8] R. Percacci, Gravity from a particle Physicists' perspective, Proc. Sci., ISFTG2009 (2009) 011.

[9] N. Cantarini, A. Ricciardo, and A. Santi, Classification of simple linearly compact Kantor triple systems over the complex numbers, J. Algebra 514, 468 (2018).

[10] P. Truini, Exceptional Lie Algebras, SU(3) and Jordan pairs, Pac. J. Math. 260, 227 (2012).

[11] A. Marrani and P. Truini, Exceptional Lie Algebras, SU(3) and Jordan pairs part 2: Zorn-type representations, J. Phys. A 47, 265202 (2014).

[12] A. Marrani and P. Truini, Exceptional Lie Algebras at the very foundations of space and time, $\mathrm{p}$ Adic Ultra. Anal. Appl. 8, 68 (2016).

[13] P. Truini, M. Rios, and A. Marrani, The magic star of exceptional periodicity, in Proceedings of the AMS 4th Mile High Conference on Nonassociative Mathematics (University of Denver, Denver, 2017).

[14] P. Truini, M. Rios, and A. Marrani, On unimodular lattices and exceptional periodicity (to be published).

[15] J. C. Baez, The Octonions, Bull. Am. Math. Soc. 39, 145 (2002).

[16] S. Catto, Exceptional projective geometries and internal symmetries, arXiv:hep-th/0302079.

[17] E. B. Vinberg, The Theory of Convex Homogeneous Cones, in Transaction of the Moscow Mathematical Society (American Mathematical Society, Providence, RI, 1965), pp. 340-403.

[18] A. Marrani and M. Rios, Higher dimensional SYM's from EP (to be published).
[19] G. T. Horowitz and L. Susskind, Bosonic M-theory, J. Math. Phys. (N.Y.) 42, 3152 (2001).

[20] E. Witten, Three-dimensional gravity revisited, arXiv:0706 .3359 .

[21] R. A. Wilson, The Leech lattice, QMUL Pure Mathematics Seminar notes, Online print.

[22] M. R. Gaberdiel, C. A. Keller, and H. Paul, Mathieu moonshine and symmetry surfing, J. Phys. A 50, 474002 (2017).

[23] I. Rudychev, E. Sezgin, and P. Sundell, Supersymmetry in dimensions beyond eleven, Nucl. Phys. B, Proc. Suppl. 68, 285 (1998).

[24] T. Banks, W. Fischler, S. H. Shenker, and L. Susskind, $M$ theory as a matrix model: A conjecture, Phys. Rev. D 55, 5112 (1997).

[25] A. Connes, Noncommutative geometry year 2000, arXiv: math/0011193.

[26] J. S. Milne, Motives-Grothendieck's dream, Online print.

[27] N. Jacobson, Structure and Representations of Jordan Algebras (American Mathematical Society, Providence, 1968), Vol. 39.

[28] R. D. Schafer, An Introduction to Nonassociative Algebras (Academic Press, New York, 1966/Dover, Mineola, N.Y., 1995).

[29] P. Truini and L. C. Biedenharn, An $E_{6} \times U(1)$ invariant quantum mechanics for a Jordan pair, J. Math. Phys. (N.Y.) 23, 1327 (1982).

[30] M. Günaydin, Lectures on spectrum generating symmetries and U-duality in supergravity, extremal black holes, quantum attractors and harmonic superspace, Springer Proc. Phys. 134, 31 (2010).

[31] J. C. Baez and J. Huerta, Division Algebras and Supersymmetry I, in Superstrings, Geometry, Topology, and $C^{*}$-Algebras, edited by R. Doran, G. Friedman, and J. Rosenberg (AMS, Providence, 2010).

[32] L. Smolin, The exceptional Jordan algebra and the matrix string, arXiv:hep-th/0104050.

[33] M. Günaydin, K. Koepsell, and H. Nicolai, Conformal and quasiconformal realizations of exceptional Lie groups, Commun. Math. Phys. 221, 57 (2001).

[34] R. Kallosh and A. Rajaraman, Vacua of M-theory and string theory, Phys. Rev. D 58, 125003 (1998). 
[35] B. de Wit, K. Peeters, J. Plefka, and A. Sevrin, The M-theory two-brane in $\mathrm{AdS}_{4} \times S^{7}$ and $\mathrm{AdS}_{7} \times S^{4}$, Phys. Lett. B 443, 153 (1998).

[36] P. Claus, Super M-brane actions in $\operatorname{adS}_{4} \times S^{7}$ and $\operatorname{adS}_{7} \times S^{4}$, Phys. Rev. D 59, 066003 (1999).

[37] H. Nishino and E. Sezgin, Supersymmetric Yang-Mills equations in $(10+2)$-dimensions, Phys. Lett. B 388, 569 (1996).

[38] H. Nishino, Supergravity in $(10+2)$ dimensions as consistent background for superstring, Phys. Lett. B 428, 85 (1998).

[39] H. Nishino, $\mathcal{N}=2$ chiral supergravity in $(10+2)$ dimensions as consistent background for super $(2+2)$ brane, Phys. Lett. B 437, 303 (1998).

[40] D. Kutasov and E. Martinec, New principles for string/ membrane unification, Nucl. Phys. B477, 652 (1996).

[41] I. Bars, Supersymmetry, p-brane duality and hidden spacetime dimensions, Phys. Rev. D 54, 5203 (1996).

[42] I. Bars and C. Kounnas, String and particle with two times, Phys. Lett. B 402, 25 (1997).

[43] L. Castellani, A locally supersymmetric $S O(10,2)$ invariant action for $D=12$ supergravity, J. High Energy Phys. 06 (2017) 061.

[44] T. Kugo and P. K. Townsend, Supersymmetry and the division Algebras, Nucl. Phys. B211, 157 (1983).

[45] R. D’Auria, S. Ferrara, M. A. Lledó, and V. S. Varadarajan, Spinor Algebras, J. Geom. Phys. 40, 101 (2001).
[46] A. Marrani, P. Truini, and M. Rios, The Magic of Being Exceptional, arXiv:1811.11208.

[47] C. T. McMullen, Dynamics on K3 surfaces: Salem numbers and Siegel disks, Online print.

[48] P. Aspinwall and D. Morrison, String Theory on K3 Surfaces, in Mirror Symmetry II, edited by B. Greene and S.-T. Yau (International Press, Cambridge, 1997), pp. 703-716.

[49] P. Jordan, J. von Neumann, and E. P. Wigner, On an Algebraic generalization of the quantum mechanical formalism, Ann. Math. (N.Y.) 35, 29 (1934).

[50] B. A. Rosenfeld, Geometrical interpretation of the compact simple Lie groups of the class E, Dokl. Akad. Nauk SSSR 106, 600 (1956).

[51] J. Tits, Buildings of Spherical Type and Finite BN-Pairs, in Lecture Notes in Mathematics Vol. 386 (Springer, Berlin, Heidelberg, 1974).

[52] K. Tent, Tits Buildings and the Model Theory of Groups, in London Mathematical Society Lecture Note Series Vol. 291 (Cambridge University Press, Cambridge, England, 2002).

[53] J. H. Conway and N. J. A. Sloane, Twenty-three constructions for the Leech lattice, Proc. R. Soc. A 381, 275 (1982).

[54] J. F. R. Duncan and S. Mack-Crane, The moonshine module for Conway's group, SIGMA 3, e10 (2015).

[55] A. Iqbal, A. Neitzke, and C. Vafa, A mysterious duality, Adv. Theor. Math. Phys. 5, 769 (2001). 